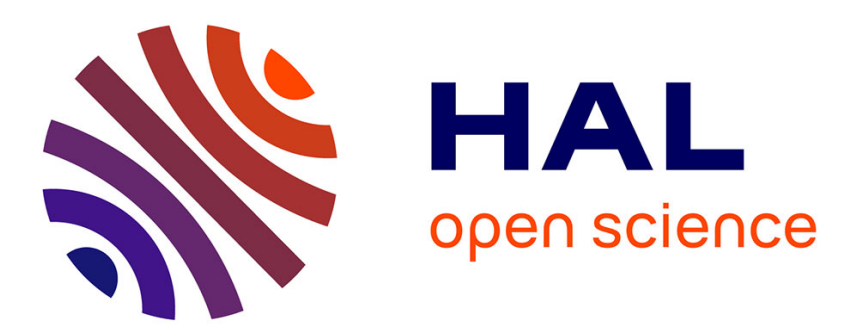

\title{
Improved Crop Classification with Rotation Knowledge using Sentinel-1 and -2 Time Series
}

Sébastien Giordano, Simon Bailly, Loic Landrieu, Nesrine Chehata

\section{To cite this version:}

Sébastien Giordano, Simon Bailly, Loic Landrieu, Nesrine Chehata. Improved Crop Classification with Rotation Knowledge using Sentinel-1 and -2 Time Series. Photogrammetric engineering and remote sensing, 2020, 86, pp.431 - 441. 10.14358/pers.86.7.431 . hal-03500509v1

\section{HAL Id: hal-03500509 \\ https://hal.science/hal-03500509v1}

Submitted on 6 Jan 2022 (v1), last revised 6 Jan 2022 (v2)

HAL is a multi-disciplinary open access archive for the deposit and dissemination of scientific research documents, whether they are published or not. The documents may come from teaching and research institutions in France or abroad, or from public or private research centers.
L'archive ouverte pluridisciplinaire HAL, est destinée au dépôt et à la diffusion de documents scientifiques de niveau recherche, publiés ou non, émanant des établissements d'enseignement et de recherche français ou étrangers, des laboratoires publics ou privés. 


\title{
Improved Crop Classification with Rotation Knowledge using Sentinel-1 and -2 Time Series
}

\author{
Sébastien Giordano, Simon Bailly, Loic Landrieu, and Nesrine Chehata
}

\begin{abstract}
Leveraging the recent availability of accurate, frequent, and multimodal (radar and optical) Sentinel-1 and -2 acquisitions, this paper investigates the automation of land parcel identification system (LPIS) crop type classification. Our approach allows for the automatic integration of temporal knowledge, i.e., crop rotations using existing parcel-based land cover databases and multi-modal Sentinel-1 and -2 time series. The temporal evolution of crop types was modeled with a linearchain conditional random field, trained with time series of multi-modal (radar and optical) satellite acquisitions and associated LPIS. Our model was tested on two study areas in France $\left(\geq 1250 \mathrm{~km}^{2}\right)$ which show different crop types, various parcel sizes, and agricultural practices: the Seine et Marne and the Alpes de Haute-Provence classified accordingly to a fine national 25-class nomenclature. We first trained a Random Forest classifier without temporal structure to achieve $89.0 \%$ overall accuracy in Seine et Marne (10 classes) and $73 \%$ in Alpes de Haute-Provence (14 classes). We then demonstrated experimentally that taking into account the temporal structure of crop rotation with our model resulted in an increase of $3 \%$ to $+5 \%$ in accuracy. This increase was especially important (+12\%) for classes which were poorly classified without using the temporal structure. A stark positive impact was also demonstrated on permanent crops, while it was fairly limited or even detrimental for annual crops.
\end{abstract}

\section{Introduction}

The Sentinel-1 and -2 satellites provide open and free acquisitions exhibiting unprecedented characteristics which are well-suited to agriculture monitoring: high temporal frequency (5-6 days), the complementary C-band Sentinel-1 radar images and multispectral Sentinel-2 images with relevant spectral bands to crop mapping, and high spatial resolution (10-20 m). In Europe, several cases of agricultural monitoring using Sentinel images have been proposed (European Commission 2016), such as observing crops (e.g., for crop area estimates, crop map products, crop phenology indicators) and controlling Common Agricultural Policy payments (e.g., for permanent grasslands, greening measures). Sentinel Images have also been used for updating and controlling the quality of the land parcel identification system (LPIS), a geographical information system on agricultural parcels, at a national scale, updated annually (Boryan, Yang, Mueller et al. 2011).

This paper introduces a tool for automated LPIS crop type classification from multi-modal Sentinel time series which incorporates knowledge from existing LPIS editions to improve its accuracy.

\section{Multi-Temporal Satellite Images for Crop Mapping}

Satellite time series are particularly well-suited for identifying different crop types, as they allow for the monitoring of the

Sébastien Giordano, Loic Landrieu, and Nesrine Chehata are with the University Paris-Est, LASTIG MATIS, IGN, ENSG, F-94160 Saint-Mandé, France.

Simon Bailly is with Wanaka, Paris, France.

Nesrine Chehata is with EA 4592, G \& E Lab, Bordeaux

INP/Bordeaux Montaigne University, France. evolution of the plant's phenology. This is particularly crucial in the growing or harvest seasons. Synthetic aperture radar (SAR) data are crucial as well, as they mitigate the effect of cloud cover.

Many studies have demonstrated the potential of multitemporal Sentinel and Landsat-8 images for crop type mapping (Palchowdhuri, Valcarce-Diňeiro, King et al. 2018; Veloso, Mermoz, Bouvet et al. 2017; Vuolo, Neuwirth, Immitzer et al. 2018; Belgiu and Csillik 2018; Ottosen, Lommen, SkjãÃÿth, 2019; Defourny, Bontemps, Bellemans et al. 2019) and the contribution of SAR time series for crop monitoring (Whelen and Siqueira, 2017; Li, Zhang, Zhang et al. 2019).

Inglada, Arias, and Tardy et al. (2015) assessed state-of-theart methods for automatic crop mapping with multi-temporal and high resolution optical images. Five different classification approaches using SPOT4 and Landsat-8 images were compared for six annual crops, over 12 different study areas, with the best results (overall accuracy) (OA $=80 \%$ ) obtained using the Random Forest classifier. In Kussul, Lemoine, and Gallego et al. (2016), Landsat-8 and Sentinel-1 time series were used on a study area in Ukraine. A pixel-based classification combined with a parcel-based regularization (majority voting) was proposed using LPIS ancillary data. An OA of $89 \%$ was reached, but only on a nomenclature comprised of six annual crops and large parcels (>250 hectares (ha)). Wagner, Narasimhan, and and Waske (2018) combined Sentinel-1 and -2 to improve land cover mapping in cloud-prone regions. Veloso, Mermoz, and Bouvet et al. (2017) showed the importance of radar data for crop mapping. More recently, the Sentinel-2 Agriculture Consortium (Sen2Agri) has led experiments at the country level (Czech Republic) using Sentinel time series for crop mapping (Sen2-Agri GISAT s.r.o. 2018). A multi-sensor (Sentinel-1, Sentinel-2) pixel-based supervised classification was performed. The LPIS was used for both learning and validation steps. Monthly cropland maps were produced with an overall accuracy greater than $80 \%$, and each land cover type had a Fscore greater than $60 \%$. In Defourny, Bontemps, Bellemans et al. (2019), three entire countries (Ukraine, Mali, and South Africa) and five local cities were mapped using Sen2Agri system. Overall accuracy values were higher than $90 \%$, and already as high as $\mathbf{8 0} \%$ midseason. However, only the five major crops were considered for each site. For the Sen2Agri framework, the nomenclature was generally limited to 5-7 classes and did not fully integrate temporal knowledge from existing data.

\section{Crop Rotation Integration}

Crop rotation knowledge can be used to improve agricultural yields (Berzsenyi, Györffy, and Lap et al. 2000) and soil quality (Karlen, Hurley, Andrews et al. 2006). Crop type prediction can also be improved using prior knowledge on crop rotations per parcel since a crop type is strongly correlated to past crop types. Modeling such temporal structures from

Photogrammetric Engineering \& Remote Sensing Vol. 86, No. 7, July 2020, pp. 431-441. 0099-1112/20/431-441

(C) 2020 American Society for Photogrammetry and Remote Sensing doi: 10.14358/PERS.86.7.431 
Sentinel image time series can lead to significant gains in classification accuracy. To take into account crop rotations in crop mapping, two issues have to be addressed:

1. How to model the crop rotations?

2. How to integrate crop rotations in a land cover classification process?

Two different approaches can be used to model rotations. The first one uses a priori agronomist expert knowledge. The second one consists in automatically learning crop rotations from the statistical analysis of past practices, as found in the LPIS annual archives. This crop rotation knowledge can then be modeled in a probabilistic framework by estimating the transition probabilities between each crop type from past years to the next. Castellazzi, Wood, Burgess et al. (2008) introduced a mathematical framework modeling such transition probabilities to predict crop rotations at the landscape scale. Few studies have yet focused on the integration of crop rotation information into classification pipelines. Osman, Inglada, and Dejoux (2015) studied early crop mapping using Markov logic, but not in combination to remote sensing observations. This model proved efficient for early crop type predictions at the beginning of the growing season, when few satellite images are available and crops are hard to distinguish. Other studies proposed to introduce a temporal structure using Hidden Markov Chains in a classification pipeline but aimed at modeling phenology instead of crop rotations (Aurdal, Huseby, Eikvil et al. 2005; Leite, Feitosa, Formaggio et al. 2011; Siachalou, Mallinis, Tsakiri-Strati 2015). Kenduiywoa, Bargiel, and Soergel (2015) modeled phenology information into a conditional random field (CRF), but the classification was performed at different dates through the year. The CRFs were used for classifying land cover classes and crop types on mono-temporal Landsat data (Roscher, Waske, Förstner 2017). Hoberg, Rottensteiner, Feitosa et al. (2015) used CRF on multitemporal and multi-scale classification for change detection.

\section{Objectives}

This paper focuses on crop type prediction using LPIS and crop rotation knowledge learned from Sentinel-1 and -2 time series. This raises three main application and methodological questions: (1) What are the respective contributions of optical and radar time series for crop type prediction? (2) How to combine crop rotation and satellite observations into a unified classification pipeline? (3) What is the contribution of the temporal structure with regard to observation-based classification?

To answer these questions, we propose a series of experiments on the areas of interest using a detailed nomenclature with 25 classes with no assumption on parcel sizes. We test the contribution of both optical and radar time series separately and jointly. We then compare the accuracy of classifying these time series each year independently and integrating the temporal structure into a probabilistic model (linear chain CRFs) representing the influence of crop rotations.

This paper is organized as follows: we present the study sites and data in the section "Sites and Material". In the section "Methodology", an observation-based classification at the parcel scale is presented, as well as a temporal-structured framework to integrate crop rotation information. Results are given and discussed in the sections "Results" and "Discussion", respectively.

\section{Sites and Material}

\section{Study Sites}

Two complementary sites were chosen in French territories. Both sites are research observatories where in-field crop type annotations are made annually. The location and characteristics of each site are provided in Figure 1 and Table 1, respectively. The site name refers to the national number of the corresponding administrative department. Site04 is located in South Eastern France, in the Alpes de HauteProvence region, in the Durance River Valley. It is a representative of Mediterranean cultivated areas. It covers $1050 \mathrm{~km}^{2}$ and is characterized by a highly variable topography, a very fragmented landscape, and a high diversity of crop types. Site77 is located near Paris, in the Seine et Marne region. It covers $233 \mathrm{~km}^{2}$. Contrary to Site04, it is characterized by a flat relief, with large parcels and a majority of cereal crops. Figure 2 shows the distribution of parcel sizes on both sites. One can see that Site04 is much more fragmented with very small parcels, while Site77 has larger parcels reaching 20 ha.

\section{Land Parcel Identification System}

In France, the land parcel identification system (LPIS) is called registre parcellaire graphique (RPG). It has been available for the whole territory since 2002. For cultivated areas, the RPG gathers the geometric information (i.e., the parcel

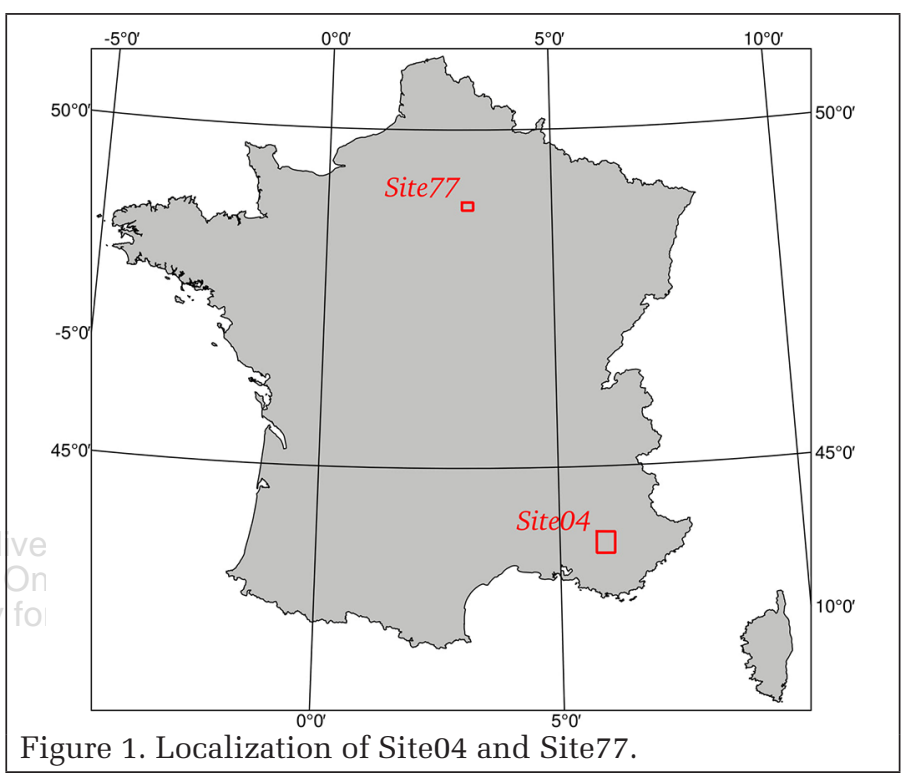

Table 1. Comparison of both study sites in terms of areas and crop types.

\begin{tabular}{lll}
\hline Class & $\begin{array}{l}\text { No. parcels- } \\
\text { Site04 }\end{array}$ & $\begin{array}{l}\text { No. parcels- } \\
\text { Site77 }\end{array}$ \\
\hline Corn & 147 & 350 \\
Barley & 517 & 158 \\
Other cereals & 2176 & 889 \\
Rapeseed & 154 & 85 \\
Sunflower & 293 & $\mathrm{X}$ \\
Other oilseeds & 116 & $\mathrm{X}$ \\
Protein (peas) & 87 & 76 \\
Fiber plants & $\mathrm{X}$ & 76 \\
Forage crops & 1215 & 46 \\
Meadows & 3652 & 725 \\
Fruit trees & 298 & 30 \\
Vineyards & 249 & $\mathrm{X}$ \\
Olive groves & 1029 & $\mathrm{X}$ \\
Aromatic crops & 1452 & $\mathrm{X}$ \\
Vegetables & 520 & 131 \\
Total no. classes & 14 & 10 \\
Total no. stable parcels & 9230 & 1902 \\
(2015-2016) & 1050 & 233 \\
Site area (km ${ }^{2}$ ) & &
\end{tabular}




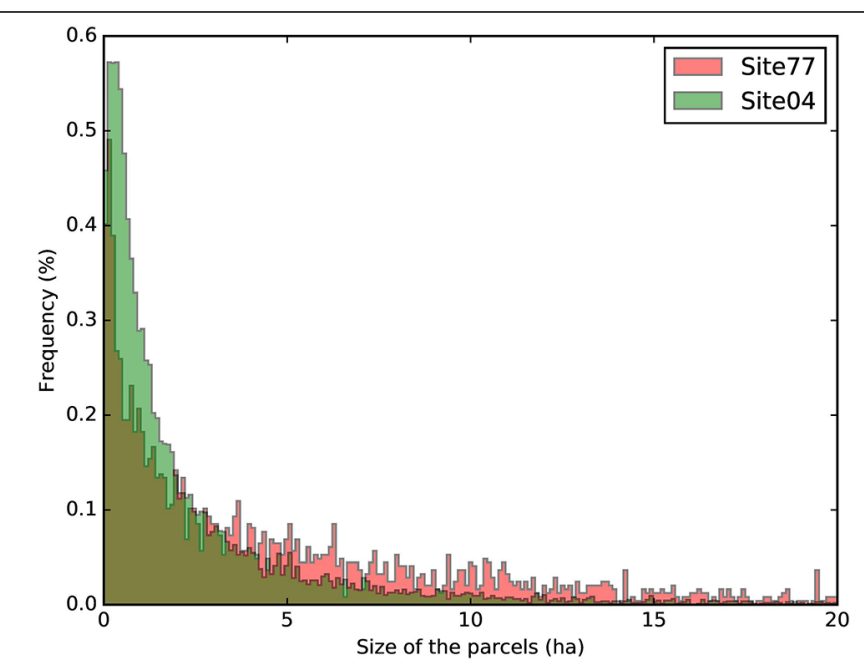

Figure 2. Normalized histogram of parcel areas for Site04 and Site77.

delimitation) and the corresponding semantic information such as the owner, the operator, the area, and the crop type.

Both geometric and semantic information are updated by the farmers annually. Until 2014, the declarations were made at a block scale that corresponds to contiguous parcels with the same operator. Since 2015, the declarations have been made at the parcel scale, which simplifies machine-learning based approaches to crop prediction. The crop type is specified among more than 300 subclasses which are organized into 25 classes. 14 and 10 of these classes are present on Site04 and Site77, respectively (cf. Table 1).

Figures 3 and 4 show the 2016 RPG edition, i.e., the ground truth data on Site04 and Site77 respectively, with the corresponding crop types. For Site04, dominant crops are: cereals $(23.8 \%)$, meadows $(30.7 \%)$, aromatic crops $(12.2 \%)$, forage crops $(10.2 \%)$, and olive groves $(8.6 \%)$. As for Site 77 , two dominant crops are present: cereals (57.7\%) and meadows $(28.3 \%)$, followed by vegetables $(5.1 \%)$. In this latter case, the data is highly imbalanced, making Site77 classification task more complex.

In this study, to be in tune with the first Sentinel-2 images availability, only the 2016 edition of parcel-based RPG was used for the training and the validation of the supervised classification model (see the section "Parcel-Wise Multi-Source Classification"). The 2015 parcels were necessary to train the temporal structured method (see the section "TemporalStructured Classification"). For learning crop rotations, only the geometrically stable blocks of parcels from 2010 to 2014 were used. The number of geometrically stable parcels for both sites is given in Table 1 .

\section{Multimodal Sentinel-1 and -2 Images}

We use both optical and radar Sentinel time-series for crop mapping. Sentinel-2 (S2) provides 10 multispectral bands for earth observation on the visible-short-wave infrared (VIS-SWIR) domain at $10 \mathrm{~m}$ and $20 \mathrm{~m}$ spatial resolution. Near infrared (NIR) and red-edge bands allow a fine characterization of crops. Sentinel-1 (S1) is a C-band SAR. The available mode on the studied sites was the Interferometric Wide (IW) mode that presents a dual polarization VV and $\mathrm{VH}$.

For the year 2016, Sentinel-2 images were automatically downloaded from the Theia platform (https://www.theialand.fr/) in tiled format, calibrated as Top of Canopy (TOC) reflectance (Hagolle, Huc, Villa Pascual, et al. 2010) and accompanied with robust cloud mask information (Hagolle, Huc, Villa Pascual, et al. 2015). The 20 m Sentinel-2 images were resampled to $10 \mathrm{~m}$ spatial resolution. Radar Sentinel-1 images were downloaded from the Peps platform (https://

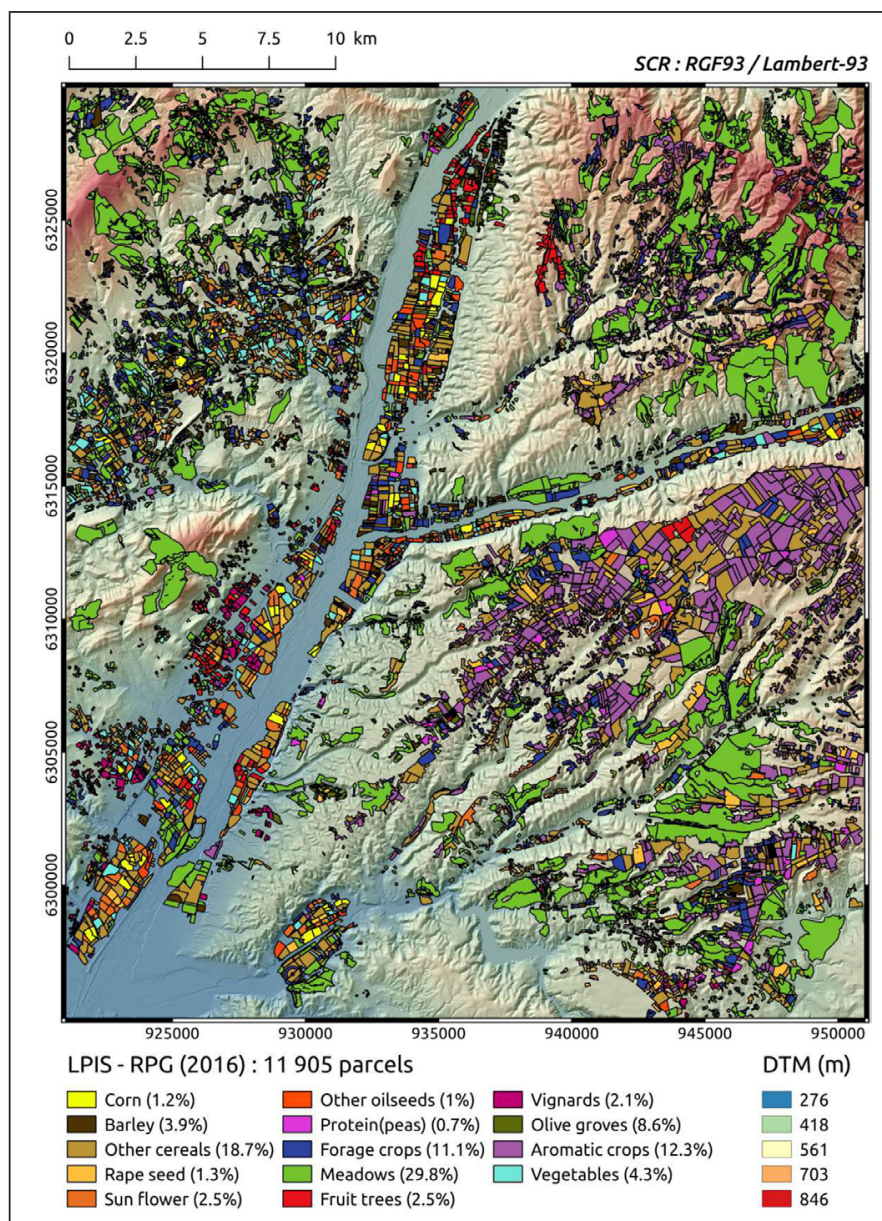

Figure 3. Site04: 2016 RPG parcel superimposed to a very high resolution Digital Terrain Model.

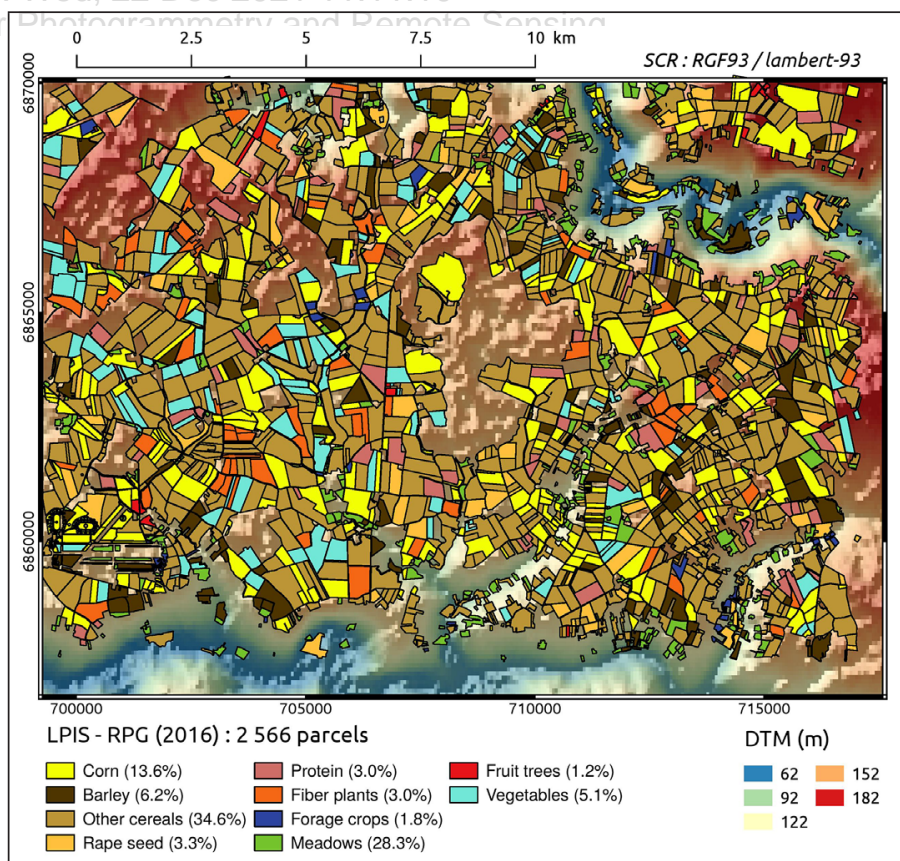

Figure 4. Site77: 2016 RPG parcel superimposed to a SRTM Digital Terrain Model.

peps.cnes.fr) in the Ground Range Detected format (GRD) which corresponds to the average of approximately five Single Look Complex acquisitions corrected by the incidence angle and resampled at $10 \mathrm{~m}$ spatial resolution. 
The total number of images is given in Table 2 and confirms the complementarity between Sentinel-1 and Sentinel-2 images. On Site77, particularly high cloud coverage led to only 12 Sentinel-2 images. In addition, S2 orbit over Site77 had many acquisition problems in 2016. On the contrary, on this site, ascendant S1 images overlap, leading to more available radar images.

\section{Sentinel Images Preprocessing}

Figure 5 illustrates the optical and radar preprocessing steps to obtain parcel-based features for the classification workflow.

The dual polarization GRD S1 images were first calibrated to $\sigma 0$ radar backscattering coefficient. The orthorectification was performed using the Shuttle Radar Topography Mission (SRTM) digital terrain model. The speckle is partly removed using a simple $5 \times 5$ Lee filter (Lee 1980). In addition to VV and VH radar features, an extra radar feature $\left(\sigma 0_{V H} / \sigma 0_{V V}\right)$ was derived. This ratio is known to be more robust to acquisition system errors or environmental factors such as soil moisture leading to a more stable temporal indicator (Veloso, Mermoz, Bouvet et al. 2017). Average and standard deviation of these three features were then computed for each date and for each parcel. The number of radar features is shown in Table 2.

Sentinel-2 images were already orthorectified and calibrated in TOC reflectance. On Site77, only 12 Sentinel-2 optical images were obtained in 2016, as shown in Figure 6 with corresponding cloud cover, whereas 23 images were available on Site04 (cf. Figure 7). The missing data (clouds) were filled using a multi-temporal spline interpolation (Inglada 2016). Average and standard deviation of the 10 spectral bands and the Normalized Difference Vegetation Index (NDVI) per optical image were then computed for each date and for each parcel. The number of optical features is shown in Table 2.

\section{Methodology}

Our method proceeds in two steps: parcel-wise classification and temporal modeling. The first step aims to predict the crop types per parcel using Sentinel time-series for each year independently. The second step integrates the temporal structure into a probabilistic structured model representing the influence of crop rotations. Modeling this temporal dependency may help correct erroneous classifications made in the first step and may also help classify ambiguous parcels by considering crops from previous years.

\section{Parcel-Wise Multi-Source Classification}

We first compute discriminative parcel-based features from satellite time-series. For each parcel, we consider all available optical and radar images for one year. We consider the average and standard deviation of each spectral feature over the pixels composing the parcel extent. We then concatenate the observations over the span of a year of acquisitions. A Random Forest classifier provides a parcel-wise prediction under the form of pseudoprobabilities.

For a given parcel $i$ and a given year $t$, we denote $X_{i}^{(t)} \in \mathrm{R}^{D}$ the tensor of combined selected features, with $D$ the selected feature size. Since we compute both mean and standard deviation for each channel at each time step, $D=2 \times C \times S$, with $C$ the number of channels, and $S$ the number of acquisitions per year. To counterbalance the over-representation of certain classes in our data sets, we set class weights inversely proportional to the square root of the number of instances in each class. This class weights are used by the random forest classifier to give more importance to rare classes and recover them more easily.

\section{Temporal-Structured Classification}

We now consider the year-by-year temporal structure of each parcel independently. We denote by $X_{i}^{(t)} \in \mathrm{R}^{T \times D}$ the sequence

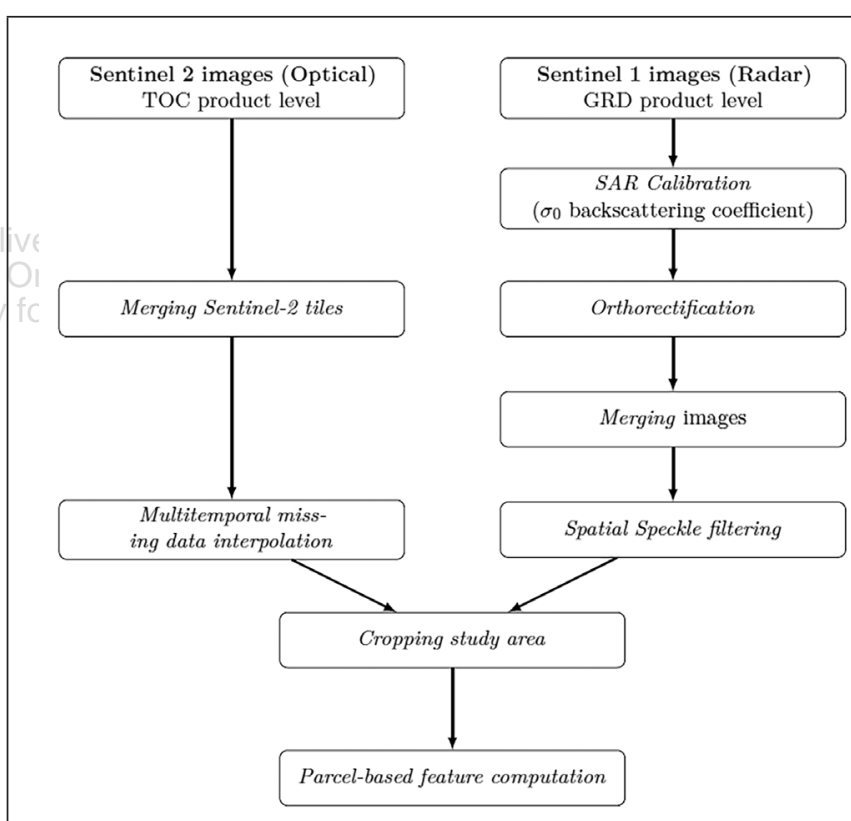

Figure 5. Sentinel-1 and -2 preprocessing steps.

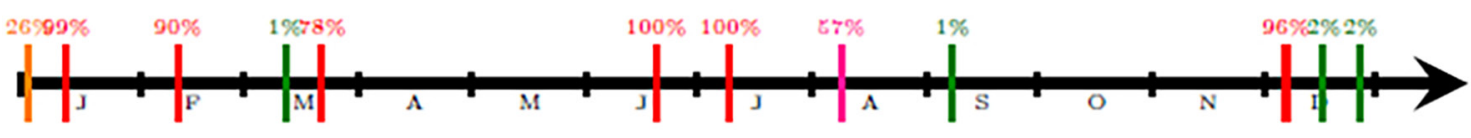

Figure 6. S2 optical images over the year 2016 and corresponding cloud cover on Site04.

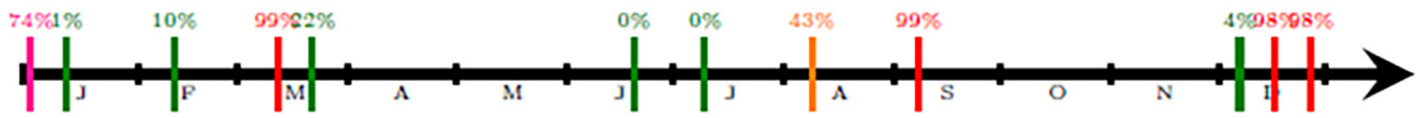

Figure 7. S2 optical images over the year 2016 and corresponding cloud cover on Site77. 
of observed features $X_{i}^{(t)} \in \mathrm{R}^{D}$ for parcel $i$ and for all years $t=1, \cdots, T$ available for training. Likewise, we denote $Y_{i} \in \mathcal{K}^{T}$ the ground truth labels for parcel $i$ for each observed year $t=1, \cdots, T$ and with $\mathcal{K}$ the set of all possible labels.

In the section "Temporal Structure", we present the graphical model chosen to capture crop rotation. In the section "Learning", we explain how the parameters of this model can be learned from previous LPIS editions. In the section "Inference", we explain how to use our model to compute prediction of the label of a parcel at a given date.

\section{Temporal Structure}

The aim of this step is to model the yearly crop rotations in order to improve crop type prediction. We model this dependency with a linear chain CRF of order $m$, as shown in Figure 8. For a parcel $i$, we model the posterior distribution $P(Z i \mid X i)$ of predicted labels $Z_{i} \in \mathcal{K}^{T}$ given the observed features $X_{i}$ as:

$$
P(Z i \mid X i)=\frac{1}{A} \exp \left(\sum_{t=1}^{T} O\left(Z_{i}^{(t)}, X_{i}\right)+\sum_{t=m+1}^{T} I\left(Z_{i}^{(t-m)}, \ldots, Z_{i}^{(t)}, X\right)\right)
$$

where $A$ is a normalizing factor, $O$ the observation potentials, and $I$ the interaction potentials, described below.

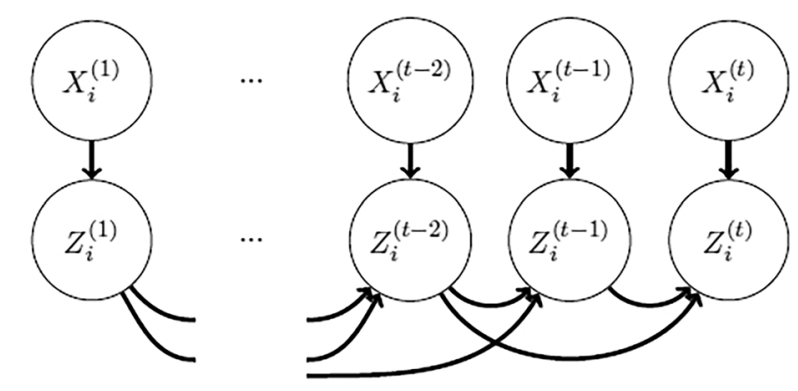

Figure 8. Graph structure of the temporal dependency at order 2.

Observation potential: The observation potential models the link between the observed features and the label of each parcel. $O\left(Z_{i}^{(t)}, X_{i}\right)$ is taken as the logarithm of $P_{R F}\left(Z_{i}^{(t)}, X_{i}\right)$, the pseudoprobability for parcel $i$ at year $t$ to be class $Z i(t)$ given by the Random Forest classifier, described in the section "Parcel-Wise Multi-Source Classification":

$$
O\left(Z_{i}^{(t)}, X_{i}\right)=\log P_{R F}\left(Z_{i}^{(t)}, X_{i}\right)
$$

Interaction potential: This potential models the temporal dependencies between the parcel's labels at a given year $t$ given the labels at the $m$ previous years. We model this potential as the logarithm of the transition probability $M\left(Z i(t-m), \cdots, Z_{i}^{(t)}\right)$ from the sequence of $m$ previous labels $Z_{i}^{(t-m)}, \ldots, Z_{i}^{(m)}$, to a label $Z_{i}^{(t)}$ at the current date (Liu, Song, Townshend et al. 2008). For the sake of simplicity, we choose a temporally homogeneous parametrization, independent of the observed features, and shared by all parcels and years:

$$
I\left(Z_{i}^{(t-m)}, \ldots, Z_{i}^{(t)}, X\right)=\log \left(M\left(Z_{i}^{(t-m)}, \ldots, Z_{i}^{(t)}\right)\right)
$$

with $M \in R^{\mathcal{K}^{m+1}}$ a tensor such that

$$
\sum_{z_{1}, \ldots, z_{m-1} \in \mathcal{K}^{m-1}} M_{z_{1}, \ldots, z_{m-1}, z_{m}}=1
$$

for all $z_{m} \in \mathcal{K}$, i.e., a stochastic tensor of order $m$. The tensor $M$ is referred to as the transition tensor.

\section{Learning}

The observation potential is obtained by training the random forest classifier. A transition tensor $\hat{M}$ can be learned from labeled data over past years. Indeed, maximizing the log-likelihood in Equation 1 with respect to

$$
z_{1}, \ldots, z_{m} \in \mathcal{K}
$$

to $M$ yields the following tensor $M$, defined for all

$$
\hat{M}_{z_{1}, \ldots, z_{m}}=\frac{N_{z_{1}, \ldots, z_{m}}}{N_{z_{1}, \ldots, z_{m-1}}}
$$

with $N_{k_{1}, \ldots, k_{m}}$ the number of sequences $k_{1}, \ldots, k_{m}$ observed in the labeled data for all parcels and all years, and $N_{k_{1}, \ldots, k_{m-1}}$ the number of sequences $k_{1}, \ldots, k_{m-1}$ observed in the first $T-1$ years, where $T$ is the total number of years available for training. Excluding the last year is necessary to ensure that $\hat{M}$ is indeed a stochastic tensor.

To account for the large size of this matrix $\left(|\mathcal{K}|^{m+1}\right)$, and to prevent numeric issues, we perform a Laplacian smoothing with $\alpha=1$ as described in Manning, Raghavan, and Schütze (2008, chapter 11.3.2)).

\section{Inference}

The aim of this step is to predict the label $Z_{i}^{(t)}$ of a given parcel at year $t$ from the observation of the current year, and knowing its labeling in the $m$ previous years. Once the random forest yielding the observation potential is trained on all available data, and the transition tensor $\hat{M}$ estimated, the prediction is given by:

$$
\left.P\left(Z_{i}^{(t)}=k \mid Z_{i}^{(t-m, \ldots, t-1)}, X_{i}\right) \propto P_{R F}\left(Z_{i}^{(t)}, X_{i}\right) \times \widehat{M}\left[Z_{i}^{(t-m)}, \ldots, Z_{i}^{(t-1)}\right]\right)
$$

and normalizing the results over $k \in \mathcal{K}$ to obtain a probability.

\section{Experimental Setup}

The random forest classifier is composed of 100 decision trees. The meta-parameters of the forest, such as the maximum number of attributes considered at each node, are chosen by $k$-fold cross-validation with $k=4$.

For the temporal structure, spatiotemporal homogeneity hypothesis allows us to estimate the transition tensor $\hat{M}$. For each study site, we use the geometrically stable parcel blocks over a period of five years (2010-2014). To decrease the number of parameters, only first order transitions were used (transition from one year to the next).

The data is randomly split equally into two distinct training and testing sets. The model is trained and validated on the training set while the quality of the model is estimated on the testing set. The OA is used to assess the general performance of the model. The F-score combines user accuracy (UA, or precision) and producer accuracy (PA, or recall) and allows estimating the per-class quality. The F-score for a class $C$ is defined as follows:

$$
\text { F-score }(C)=2 * \frac{U A_{c^{*}} P A_{c}}{U A_{c}+P A_{c}}
$$

To sum up this information, the F-scores are averaged, with and without weighting by each class cardinality. The weighted F-scores reduce biases due to imbalanced data. The results are averaged over 10 runs.

\section{Results}

The results are presented on two distinct agricultural sites $\left(\geq 1250 \mathrm{~km}^{2}\right.$ ), showing different crop types with highly 
imbalanced data, various parcel sizes, different agricultural practices, and acquired by different Sentinel image distributions. Prediction accuracies are presented using different feature combinations and both with and without temporal modeling. The impact of parcel size on the classification accuracy is also studied.

\section{Transition Matrix Assessment}

Figure 9 shows the estimated transitions between crop types as Hinton diagrams for both sites. First order transitions are normalized by the number of parcels of the initial class (year $n-1)$ which ensures it to be nonsensitive to imbalanced data. Besides, in case of missing crops, a smoothing is processed to avoid zeros in the transition matrix (cf. the section "Learning”). This smoothing may lead to some biases on minor classes but do not impact the results.

On Site04, the most probable transitions are to and from permanent crops, such as olive groves, vineyards, orchards, permanent meadows, and fruit trees reaching 98.34\%, $93.87 \%, 92.72 \%, 91.89 \%$, and $84.23 \%$, respectively. From Figure 9, we can observe that the standard rotation patterns of annual crops are generally not applied in this area. For instance, the rapeseed, proteins, and sunflowers have probabilities of $76.53 \%, 66.78 \%$, and $64.25 \%$, respectively to be transformed to other cereals the following year.

On Site77, more transitions are observed for the annual crops. Agricultural rules for annual crop rotations seem to be better followed in this area. The rapeseed and proteins have probabilities of $97.09 \%$ and $94.85 \%$, respectively, to be transformed to other cereals the following year. Indeed, rapeseed $\rightarrow$ winter wheat (in other cereals) $\rightarrow$ barley is a well-known three-year rotation for farmers of this area. Permanent crops such as meadows and fruit trees have a probability of being carried over the next year of $94.45 \%$ and $81.39 \%$, respectively.

\section{Optical versus Radar Sentinel Time Series}

Overall accuracy and F-scores, using different configurations of optical and radar data, are displayed in Tables 3 and 4 for Site04 and Site77, respectively.

As reported in Site04 (cf. Table 3), optical data lead to better results than radar data $(+9 \%$ for OA and $+10 \%$ for weighted F-score). This may be explained by a low cloud cover in this area (Figure 7) and a finer native resolution of optical imagery that is more suited to small parcel sizes. Table 3 confirms that optical and radar combination led to the best results when not modeling the temporal structure.
Contrary to the previous site, on Site77 (cf. Table 4), radar attributes improved the results of optical ones by $7 \%$, achieving an overall accuracy of $89 \%$. This can be explained by a combination of frequent acquisition problems and a high cloud cover in 2016, leading to many missing optical Sentinel-2 data (cf. Figure 6). In addition, the parcels on Site77 are larger and thus more compatible with radar Sentinel-1 image spatial resolution. Consequently, using radar imagery solely led to similar results when combining optical and radar attributes.

Weighted F-scores, using combined radar and optical images, reached $88 \%$ and $71 \%$ on Site77 and Site04, respectively. The crop type mappings and prediction errors are illustrated on Figures 10, 11, 12, and 13 for Site04 and Site77, respectively for test parcels.

\section{Impact of Temporal Structure}

Table 5 and Table 6 display the F-score, the user, and producer accuracy measures per class for both approaches with and without temporal modeling using combined radar and optical images on Site04 and Site77, respectively. On Site77, from Table 6, one can see that high F-scores are obtained for annual

Table 3. Site04-Global prediction accuracies, using optical and radar imagery.

\begin{tabular}{lccc}
\hline Config & OA & F-score & Weighted F-score \\
\hline No temporal modeling & & & \\
Radar & 0.64 & 0.59 & 0.61 \\
Optical & $\mathbf{0 . 7 3}$ & 0.67 & $\mathbf{0 . 7 1}$ \\
Radar + Optical & $\mathbf{0 . 7 3}$ & $\mathbf{0 . 6 8}$ & $\mathbf{0 . 7 1}$ \\
\hline Temporal modeling & & & \\
Radar & 0.76 & 0.60 & 0.7 \\
Optical & 0.78 & 0.63 & $\mathbf{0 . 7 2}$ \\
Radar + Optical & $\mathbf{0 . 7 8}$ & $\mathbf{0 . 6 4}$ & $\mathbf{0 . 7 2}$ \\
\hline
\end{tabular}

Table 4. Site77-Global prediction accuracy, using optical and radar imagery.

\begin{tabular}{lccc}
\hline Config & OA & F-score & Weighted F-score \\
\hline No temporal modeling & \multicolumn{2}{c}{} \\
Radar & $\mathbf{0 . 8 9}$ & 0.73 & \\
Optical & 0.82 & 0.62 & $\mathbf{0 . 8 8}$ \\
Radar + Optical & $\mathbf{0 . 8 9}$ & $\mathbf{0 . 7 4}$ & 0.81 \\
Temporal modeling & & & $\mathbf{0 . 8 8}$ \\
Radar & $\mathbf{0 . 9 2}$ & $\mathbf{0 . 7 8}$ & $\mathbf{0 . 9 1}$ \\
Optical & 0.87 & 0.67 & 0.85 \\
Radar + Optical & $\mathbf{0 . 9 2}$ & 0.76 & $\mathbf{0 . 9 1}$ \\
\hline
\end{tabular}

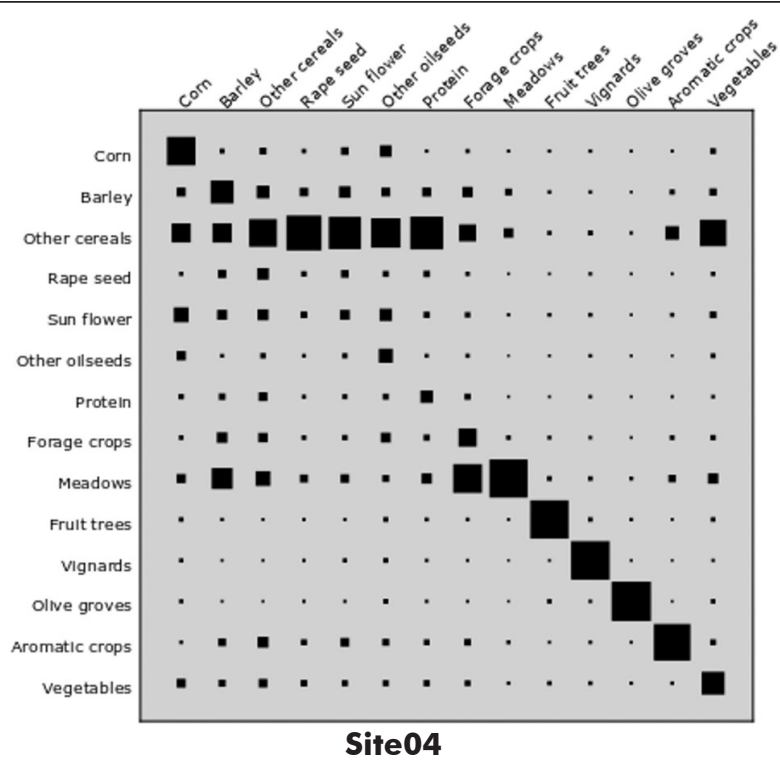

Site04

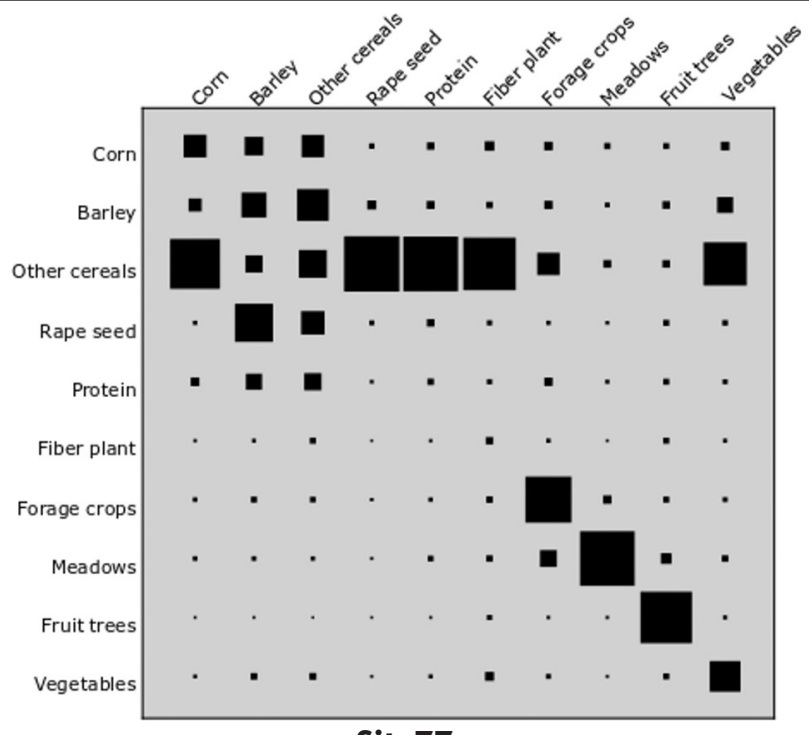

Site77

Figure 9. Representation of the transition matrices with a Hinton diagram. 


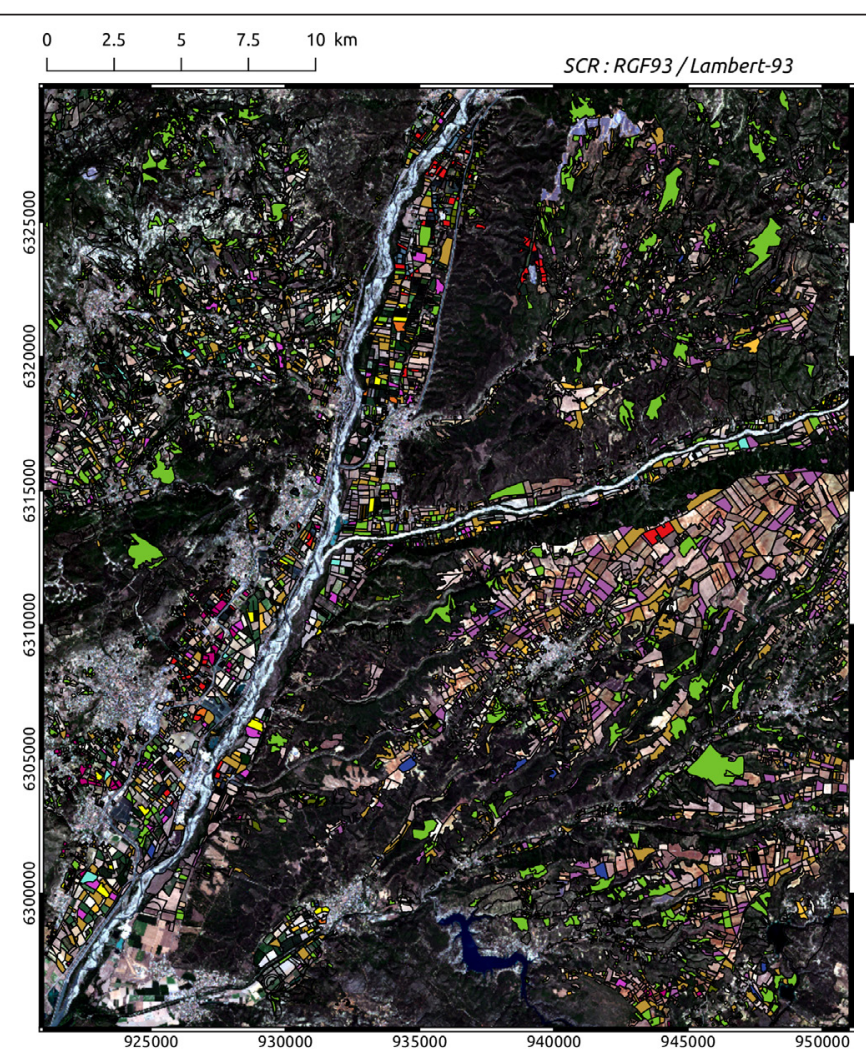

2016 Prediction

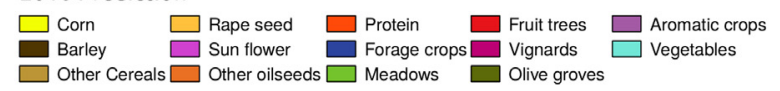

Figure 10. Site04: Crop type predictions.

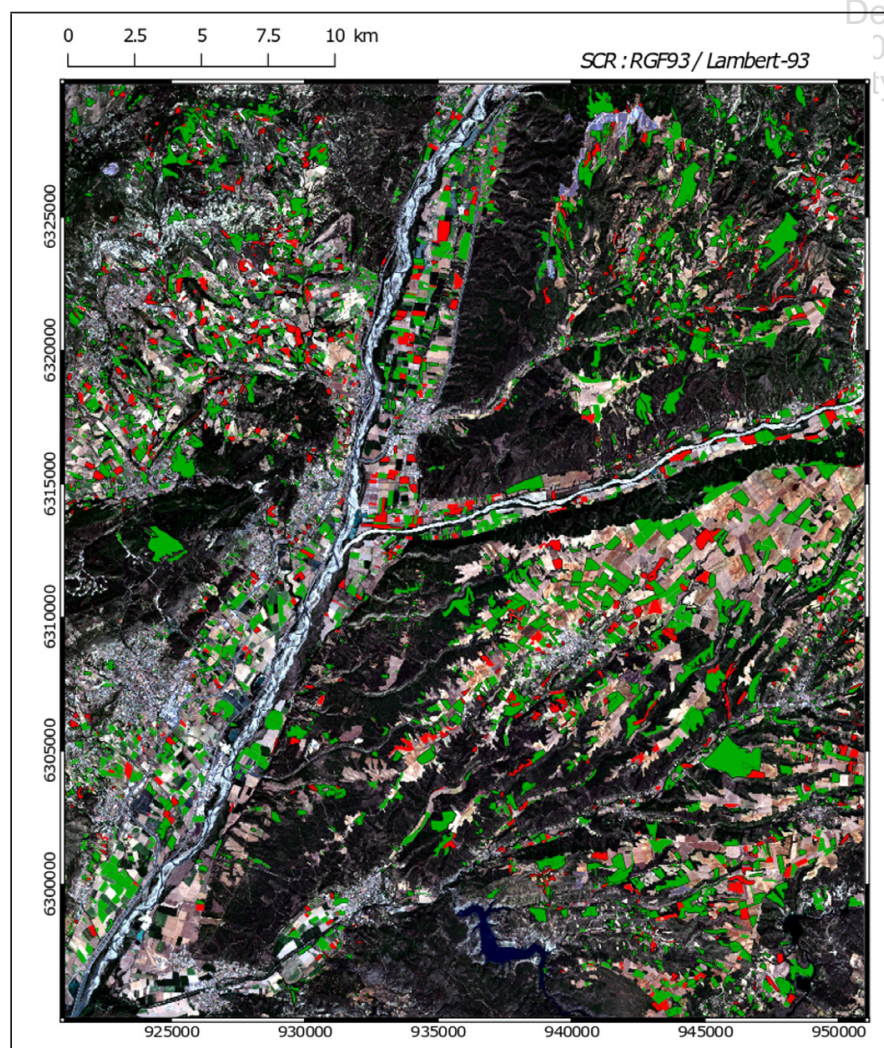

2016 Prediction errors

error ok

Figure 11. Site04: Prediction errors. crops (corn (94\%), barley (90\%), other cereals (95\%), rapeseed $(96 \%), \ldots)$. However, forage crops and fruit trees were not identified by the classifier and were instead classified as meadows (cf. Table 8). Forage crops class is harder to classify using only satellite observations since it is more a land use class than a land cover class, based on agricultural knowledge about the destination of cereal crops. Table 8 shows that the meadows class is often confused with other classes, particularly fruit trees and other cereals. Meadows are composed of trees and bare soils and have a low volumetric radar response that make them indistinguishable from other cereals.

The quality of the prediction is lower for Site04, with a weighted F-scores varying between $61 \%$ and $71 \%$. This is due to the presence of more classes (14), small size of parcels, and highly imbalanced classes where meadows and other cereals represented almost $50 \%$ of the area, as shown in Figure 3. Similar to Site77, the confusion matrix for combined optical and radar data (Table 7) shows that most ambiguities occurred on meadows classes, other cereals, and forage crops. Besides, aromatic crops represented $12.3 \%$ of the area and proved hard to classify correctly.

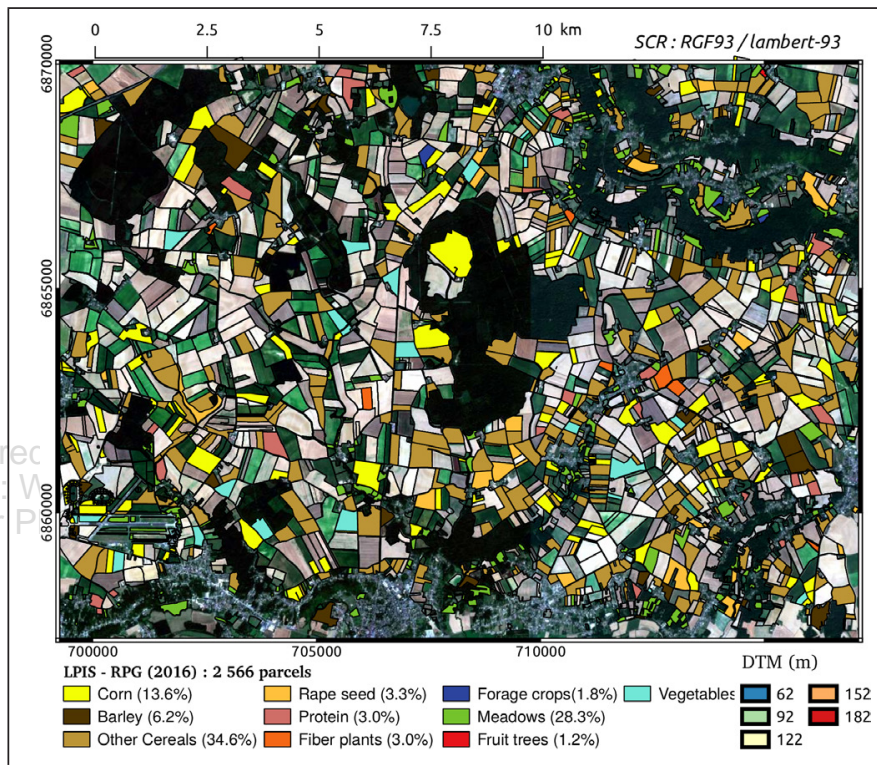

Figure 12. Site77: Crop type predictions.

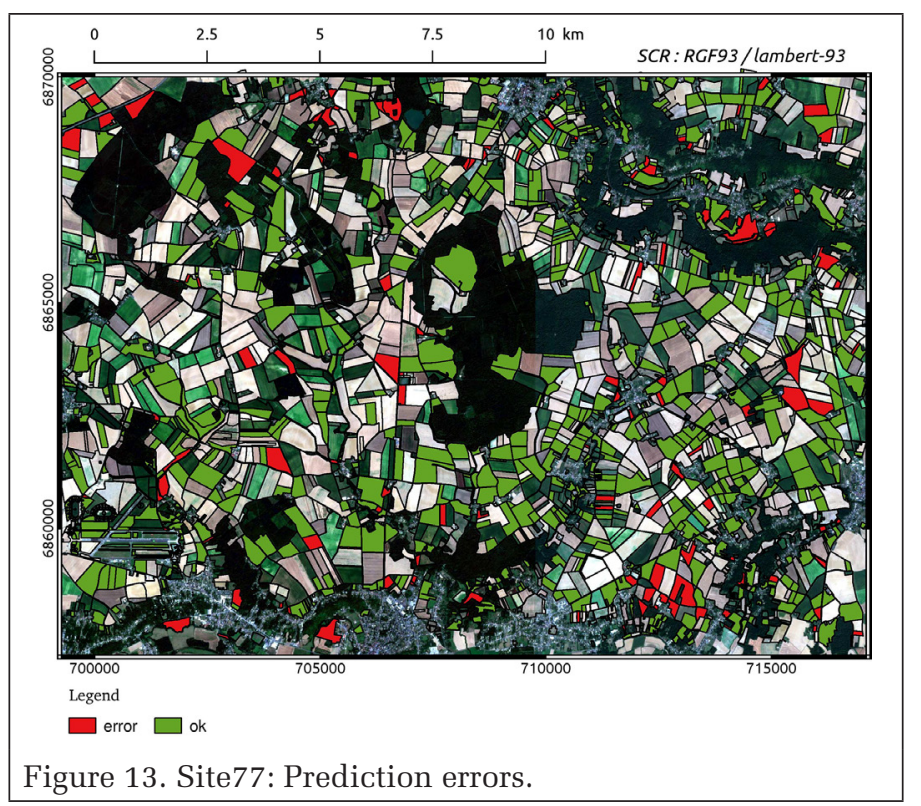


Table 5. Site04-Effect of temporal modeling on classification metrics, using combined radar and optical attributes.

\begin{tabular}{lccc|ccc}
\hline & \multicolumn{2}{c}{ No temporal modeling } & \multicolumn{3}{c}{ Temporal modeling } \\
Class & \multicolumn{2}{c}{ User. } & Proc. & \multicolumn{4}{c}{ User. } & Proc. \\
\hline Corn & 0.89 & 0.95 & 0.83 & 0.78 & 0.84 & 0.73 \\
Barley & 0.40 & 0.85 & 0.26 & 0.18 & 0.67 & 0.11 \\
Other cereals & 0.85 & 0.83 & 0.86 & 0.78 & 0.73 & 0.84 \\
Rapeseed & 0.92 & 1 & 0.86 & 0.71 & 1 & 0.55 \\
Sunflower & 0.79 & 0.78 & 0.79 & 0.71 & 0.75 & 0.68 \\
Other oilseeds & 0.57 & 0.74 & 0.46 & 0.41 & 1 & 0.26 \\
Protein & 0.49 & 1 & 0.32 & 0 & 0.07 & 0 \\
Forage crops & 0.47 & 0.73 & 0.34 & $\mathbf{0 . 1}$ & 0.88 & 0.05 \\
Meadows & 0.76 & 0.67 & 0.87 & $\mathbf{0 . 8 0}$ & 0.67 & 0.98 \\
Fruit trees & 0.61 & 0.86 & 0.47 & $\mathbf{0 . 9 7}$ & 0.96 & 0.97 \\
Vineyards & 0.69 & 0.74 & 0.65 & $\mathbf{0 . 9 9}$ & 0.99 & 0.98 \\
Olive groves & 0.74 & 0.82 & 0.67 & $\mathbf{0 . 9 9}$ & 0.99 & 0.99 \\
Aromatic crops & 0.74 & 0.65 & 0.86 & $\mathbf{0 . 9 3}$ & 0.94 & 0.92 \\
Vegetables & 0.61 & 0.72 & 0.53 & $\mathbf{0 . 6 7}$ & 0.9 & 0.54 \\
\hline
\end{tabular}

Table 6. Site77-Effect of temporal modeling on accuracy metrics, using combined radar and optical attributes.

\begin{tabular}{lccc|ccc}
\hline & \multicolumn{3}{c}{ No temporal modeling } & \multicolumn{3}{c}{ Temporal modeling } \\
Class & & User. & Proc. & & User. & Proc. \\
& F-score & Acc & Acc & F-score & Acc & Acc \\
\hline Corn & $\mathbf{0 . 9 4}$ & 0.93 & 0.95 & 0.88 & 0.83 & 0.93 \\
Barley & $\mathbf{0 . 9 0}$ & 0.94 & 0.86 & 0.82 & 0.78 & 0.85 \\
Other cereals & 0.95 & 0.96 & 0.94 & $\mathbf{0 . 9 5}$ & 0.94 & 0.97 \\
Rapeseed & 0.96 & 0.97 & 0.94 & $\mathbf{0 . 9 7}$ & 0.98 & 0.95 \\
Protein & 0.95 & 0.93 & 0.97 & $\mathbf{0 . 9 5}$ & 0.97 & 0.94 \\
Fiber plants & $\mathbf{0 . 9 7}$ & 1 & 0.95 & 0 & 0.1 & 0 \\
Forage crops & 0 & 0.1 & 0 & $\mathbf{0 . 7 0}$ & 0.78 & 0.65 \\
Meadows & 0.87 & 0.81 & 0.93 & $\mathbf{0 . 9 5}$ & 0.94 & 0.97 \\
Fruit trees & 0.01 & 0.1 & 0 & $\mathbf{0 . 9 4}$ & 1 & 0.89 \\
Vegetables & $\mathbf{0 . 8 9}$ & 0.91 & 0.88 & 0.45 & 0.97 & 0.30 \\
\hline
\end{tabular}

Impact of Parcel Size

Figures 14 and 15 show the impact of parcel size on parcelbased classification without temporal modeling on both sites. For Site04, when keeping only large parcels (area $>3$ ha), overall accuracy improves by $15 \%, 5.9 \%$, and $8.7 \%$ for radar, opti$\mathrm{cal}$, and combined optical/radar attributes, respectively (Figure 14). Indeed, due to the limited spatial resolution of Sentinel-1 images, radar attributes are less robust on small parcel sizes. When considering parcels larger than $>3$ ha, radar images achieve similar results as optical images, with an overall accuracy of $79.3 \%$. Finally, combining optical and radar data lead to better results in all cases, and especially for large parcels.

As for Site77, similarly to Site04, when considering only parcels larger than $>3$ ha, the overall accuracy is greatly improved by radar attributes (+ $8.1 \%$ reaching $97.1 \%$ ), which confirms that radar images are less robust for smaller parcels.

\section{Discussion}

In the following, we will compare the contribution of optical and Radar Sentinel time series and the impact of parcel size. We then detail the impact of modeling the temporal structure and finally explain the impact of the site characteristics on the results.

\section{Classification Without Temporal Modeling}

First, we will compare the results of both sites using parcelbased crop type prediction based on image observations only (without temporal modeling).

\section{Optical versus Radar Sentinel Time Series}

Depending on the image distribution and the cloud cover, optical data may not lead to good results for crop type mapping. In our case, temporal missing data interpolation was used, which led to some uncertainties and decreased the classification accuracy. On the other hand, radar data are less robust for small parcels $(\leq 1.5 \mathrm{ha})$. However, this issue can be reduced by refining the preprocessing framework of radar data.

Table 7. Site04-Confusion matrices using combined optical and radar attributes. metry and Remote Sensing

\begin{tabular}{|c|c|c|c|c|c|c|c|c|c|c|c|c|c|c|}
\hline & \multicolumn{14}{|c|}{ Ground truth } \\
\hline & Corn & Barley & $\begin{array}{c}0 . \\
\text { cereals }\end{array}$ & Rapeseed & Sunflower & $\begin{array}{c}\text { O. } \\
\text { oilseeds }\end{array}$ & Protein & $\begin{array}{l}\text { Forage } \\
\text { crops }\end{array}$ & Meadows & $\begin{array}{l}\text { Fruit } \\
\text { trees }\end{array}$ & Vineyards & $\begin{array}{l}\text { Olive } \\
\text { groves }\end{array}$ & $\begin{array}{l}\text { Arom. } \\
\text { crops }\end{array}$ & Vegetables \\
\hline \multicolumn{15}{|l|}{ Classification } \\
\hline \multicolumn{15}{|c|}{ Optical and radar without temporal modeling } \\
\hline Corn & 31 & - & - & - & 1 & 2 & - & - & - & - & 1 & - & - & 3 \\
\hline Barley & - & 47 & 9 & - & - & - & - & 3 & 37 & - & - & - & 13 & - \\
\hline O. cereals & - & 5 & 684 & - & 5 & 1 & - & 5 & 59 & - & - & 4 & 25 & 1 \\
\hline Rapeseed & - & - & 1 & 56 & - & - & - & - & 10 & - & - & - & - & - \\
\hline Sunflower & - & - & 1 & - & 78 & - & - & - & 2 & - & - & - & 13 & 4 \\
\hline O. oilseeds & 1 & - & - & - & 7 & 18 & - & - & 4 & - & 1 & - & 2 & 7 \\
\hline Protein & - & 2 & 6 & - & - & - & 10 & 1 & 7 & - & - & - & 2 & - \\
\hline Forage crops & - & - & 18 & - & 1 & - & - & 173 & 239 & 6 & 3 & 9 & 64 & 2 \\
\hline Meadows & - & 3 & 16 & - & 3 & 1 & - & 47 & 1294 & - & 9 & 27 & 77 & 3 \\
\hline Fruit trees & 1 & - & 1 & - & - & - & - & - & 52 & 56 & 1 & 9 & 5 & - \\
\hline Vineyards & - & - & - & - & - & - & - & - & 18 & - & 74 & 4 & 19 & - \\
\hline Olive groves & - & - & 4 & - & - & 1 & - & 1 & 113 & 1 & 6 & 311 & 26 & 1 \\
\hline Arom crops & - & - & 1 & - & - & - & - & - & 64 & - & 2 & 6 & 480 & 2 \\
\hline Vegetables & - & - & 2 & - & 6 & 4 & - & 2 & 18 & - & 4 & 6 & 9 & 60 \\
\hline \multicolumn{15}{|c|}{ Structured optical and radar } \\
\hline Corn & 28 & - & 7 & - & 1 & - & - & - & 1 & - & - & - & 1 & - \\
\hline Barley & - & 20 & 123 & - & - & - & - & - & 47 & - & - & - & - & - \\
\hline O. cereals & 1 & 1 & 676 & - & 2 & - & - & - & 102 & - & - & - & 6 & 1 \\
\hline Rapeseed & - & 5 & 21 & 35 & - & - & - & - & 6 & - & - & - & - & - \\
\hline Sunflower & - & - & 6 & - & 67 & - & - & 1 & 13 & - & - & - & 8 & 3 \\
\hline O. oilseeds & 4 & - & 4 & - & 7 & 10 & - & - & 14 & - & - & - & - & 1 \\
\hline Protein & - & 2 & 21 & - & - & - & - & - & 5 & - & - & - & - & - \\
\hline Forage crops & 1 & - & 15 & - & 3 & - & - & 25 & 461 & - & - & - & 9 & 1 \\
\hline Meadows & - & 1 & 16 & - & 1 & - & - & 2 & 1456 & - & - & 1 & 3 & - \\
\hline Fruit trees & - & - & - & - & - & - & - & - & 2 & 122 & 1 & - & - & - \\
\hline Vineyards & - & - & - & - & - & - & - & - & 3 & - & 112 & - & - & - \\
\hline Olive groves & - & - & 1 & - & - & - & - & - & 3 & 2 & - & 458 & - & - \\
\hline Arom crops & - & 1 & 17 & - & - & - & - & - & 26 & - & - & - & 511 & - \\
\hline Vegetables & - & - & 20 & - & 10 & - & - & - & 16 & 3 & - & - & 4 & 58 \\
\hline
\end{tabular}


These results confirm that combining optical and radar data ensures higher crop type prediction accuracy, and lead to more robust prediction, independently from the study site.

\section{Impact of Parcel Size}

Weighted F-scores on both sites are highly dependent on parcel sizes as detailed hereby. For both sites, when keeping only large parcels (area $>3 \mathrm{ha}$ ), overall accuracies are improved by radar attributes $(+15 \%$ and $+8.1 \%$ for Site0 4 and Site77, respectively). Indeed, due to the limited spatial resolution of Sentinel-1 images, radar attributes are less robust on small parcel sizes.

In order to make radar data more robust to parcel of limited sizes, some improvements could be undertaken on radar data preprocessing. To this end, we used speckle filtering (Lee 1980) on a restricted local neighborhood $(5 \times 5)$. This is suitable for large parcels as the radar scattering coefficients are averaged afterwards at the parcel level. However, when the parcel area is too small with respect to the Sentinel-1 spatial resolution, this method is no longer suitable. Adaptive radar speckle filtering to small objects should be investigated (Deledalle, Denis, Tupin et al. 2015).

For optical attributes, the relation between parcel size and accuracy is less pronounced. Many factors may impact the overall accuracy such as cloud cover, data imbalance, and the parcel size. Indeed, some classes are more represented in small parcels (0.5-1.5 ha) and are well identified, such as rapeseed, protein, meadows, and fiber plants. Removing these small parcels may decrease the overall accuracy.

Combining optical and radar data lead to better results in all cases, and especially for large parcels. Finally, this sensitivity study confirms the robustness of combined radar and optical data to the parcel size.

\section{Impact of Temporal Structure}

The modeling of temporal structure, i.e., crop rotation modeling, improved the global prediction accuracies on both sites. On Site04 (cf. Table 5), temporally-structured classification improved the overall accuracy and the weighted F-score by $5 \%$ and $1 \%$, respectively. When considering only radar data,

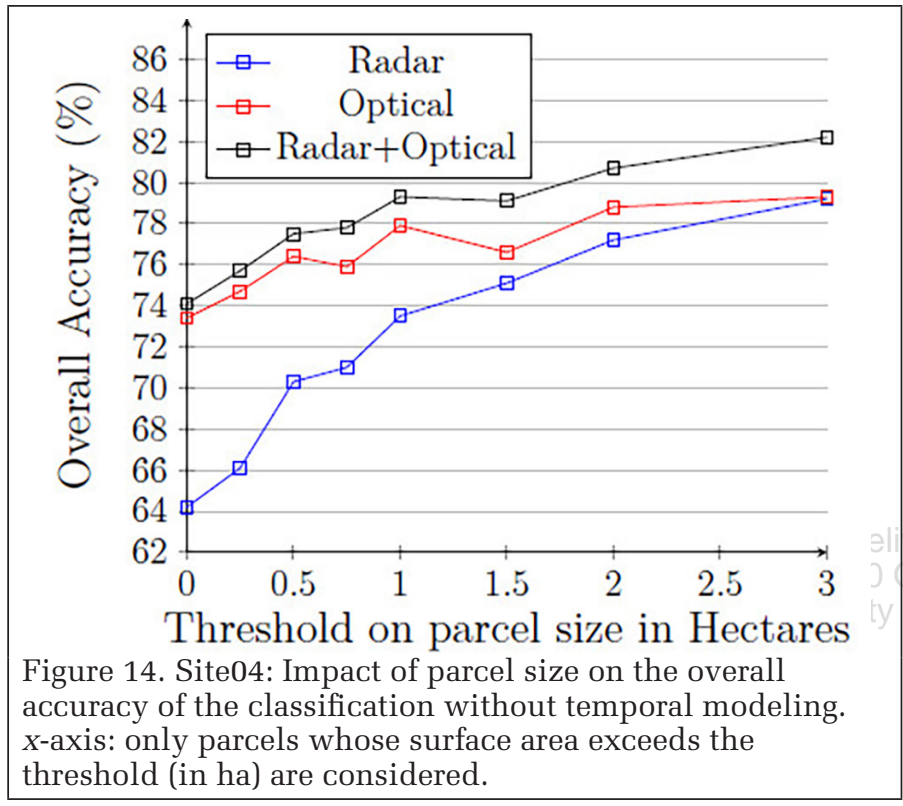

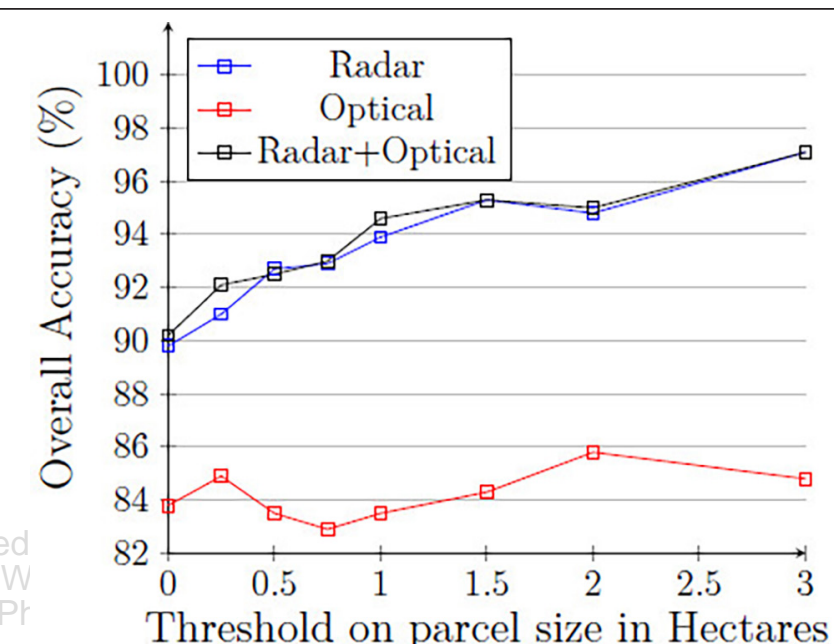

Figure 15. Site77: Impact of parcel size on the overall accuracy of the classification without temporal modeling; $x$-axis: only parcels whose surface area exceeds the threshold (in ha) are considered.

Table 8. Site77-Confusion matrices using combined optical and radar attributes.

\begin{tabular}{|c|c|c|c|c|c|c|c|c|c|c|}
\hline & \multicolumn{10}{|c|}{ Ground truth } \\
\hline & Corn & Barley & O. cereals & Rapeseed & Protein & $\begin{array}{l}\text { Fiber } \\
\text { plants }\end{array}$ & $\begin{array}{c}\text { Forage } \\
\text { crops }\end{array}$ & Meadows & $\begin{array}{l}\text { Fruit } \\
\text { trees }\end{array}$ & Vegetables \\
\hline \multicolumn{11}{|l|}{ Classification } \\
\hline \multicolumn{11}{|c|}{ Optical and radar without temporal modeling } \\
\hline Corn & 119 & - & - & - & - & - & - & 6 & - & - \\
\hline Barley & 1 & 48 & 2 & - & 1 & - & - & 3 & - & - \\
\hline O. cereals & - & 2 & 309 & - & - & - & - & 19 & - & 1 \\
\hline Rapeseed & - & - & - & 38 & - & - & - & 3 & - & - \\
\hline Protein & - & - & - & - & 27 & - & - & 1 & - & - \\
\hline Fiber plants & - & - & - & - & 1 & 9 & - & - & - & - \\
\hline Forage crops & - & - & 1 & - & - & - & - & 20 & - & - \\
\hline Meadows & 10 & 1 & 9 & 1 & - & - & - & 276 & - & 1 \\
\hline Fruit trees & - & - & - & - & - & - & - & 12 & - & - \\
\hline Vegetables & 1 & - & 1 & - & - & - & - & 1 & - & 27 \\
\hline \multicolumn{11}{|c|}{ Structured optical and radar } \\
\hline Corn & 118 & 1 & 1 & - & - & - & 3 & 2 & - & - \\
\hline Barley & 1 & 48 & 5 & - & - & - & - & 1 & - & - \\
\hline O. cereals & - & 2 & 321 & 1 & - & - & - & 7 & - & - \\
\hline Rapeseed & - & - & 1 & 39 & - & - & - & 1 & - & - \\
\hline Protein & - & - & 1 & - & 27 & - & - & - & - & - \\
\hline Fiber plants & 1 & 8 & - & - & 1 & - & - & - & - & - \\
\hline Forage crops & - & 1 & 2 & - & - & - & 13 & 5 & - & - \\
\hline Meadows & 4 & - & 4 & - & - & - & 1 & 289 & - & - \\
\hline Fruit trees & - & - & - & - & - & - & - & - & 12 & - \\
\hline Vegetables & 19 & - & 2 & - & - & - & - & - & - & 9 \\
\hline
\end{tabular}


unstructured accuracies were very low, with an overall accuracy of $64 \%$ and a weighted F-score of $61 \%$. The temporal modeling approach improved corresponding OA and weighted F-score by $12 \%$ and $9 \%$, respectively, confirming the contribution of temporal structure even if the accuracy of the parcel-wise prediction was low. As for Site77, the structured approach slightly improved the weighted F-scores by $3 \%$, $4 \%$, and $3 \%$ for radar, optical, and combined optical/radar attributes, respectively. The contribution of temporal structure is lower than that in Site04, as the initial parcel-wise accuracies were already high (weighted F-scores $>0.88$ ).

As seen in Table 5, temporal structure modeling significantly improved per-class accuracies of permanent crops (fruit trees $+36 \%$, vineyards $+30 \%$, olive groves $+25 \%$, aromatic groves $+19 \%$ for Site04 ), which reached F-scores higher than $93 \%$. The meadows class F-score was improved by $4 \%$. These results were expected since the permanent crops have the highest transition probability as shown in the section "Transition Matrix Assessment". However, F-scores of annual crops classes decreased when using temporal structure; only slightly so for corn $(-11 \%)$, other cereals $(-7 \%)$, and sunflowers $(-8 \%)$, but rapeseed $(-21 \%)$, barley $(-22 \%)$, other oilseeds $(-16 \%)$, protein $(-49 \%)$, and forage crops $(-46 \%)$ are significantly more often misclassified (cf. Table 7). This may be explained, in our opinion, by two facts: first, the crop rotations are modeled by a temporal regularization between the observation-based term (classification without temporal structure) and the crop transition probabilities. The prediction is a trade-off between both data and regularization terms. If the data-term is high (good prediction with observations), adding crop rotation information does not impact the results significantly (as for corn, rapeseed, and sunflower classes). Second, this may be due to the fact that the first order transitions of annual crops are less stable and highly variable with agricultural practices and operators in this area. Hence, temporal modeling does not add useful information and may even wrongly correct an initially correct parcel-wise prediction.

As for Site77, similarly to Site04, the best improvements occur on permanent crops, such as meadows and fruit trees (cf. Table 5). Moreover, the temporal structure improved the prediction of some annual crops, such as other cereals, rapeseeds, and proteins since they have a high first order transition probability to other cereals. The prediction of forage crops is also highly improved using crop rotations information.

On both sites, including rotation knowledge improved the overall accuracy of crop classification. The proposed model is a trade-off between observation-based classification and temporal regularization using learned rotation knowledge. If the precision of observation-based classification is already high and the transition patterns inconclusive or poorly followed, integrating rotation knowledge may decrease the accuracy. However, ambiguous observation-based prediction can be improved by modeling the temporal structure, especially if the temporal aspect is very influential, as with permanent crops or crops alternating with other cereals. The detrimental effect of temporal modeling on some annual crops were can also be explained by the limited availability LPIS (only one edition, 2016, was available at the time), which might not be sufficient to model crop rotation schemes occurring over two or three years. Indeed, in this paper, only first order crop rotation was modeled. However, our approach could be straightforwardly extended to rotations over multiple years, provided more data is available. Further tests should be processed with a newer LPIS edition and over larger areas in order to assess the effect of modeling the temporal structure.

\section{Conclusion and Perspectives}

This study focused on improving the automatic prediction of crop types using Sentinel-1 and -2 time series and learned rotation knowledge. This study demonstrated the efficiency of multi-temporal and multi-modal Sentinel (optical and radar) images for crop type classification using a fine nomenclature (>10 classes) and without filtering small parcels. The joint use of optical and radar features ensured more stable and accurate results. However, results varied highly depending on sites depending on cloud cover, crop types, and parcel size.

We modeled the temporal structure (i.e., crop knowledge) with conditional random fields and automatically learning the probability of crop rotations from previous LPIS editions. This rotation knowledge markedly improved the prediction of crop types. However, while a positive impact is demonstrated on permanent crops using first order crop transitions; this impact is fairly limited or even detrimental for some annual crops. Higher transition orders should be investigated to confirm the interest of temporal structure for annual crops and larger areas with more representative classes should be used. Finally, thanks to the growing volume of available LPIS data and the free availability of numerous Sentinel images, deep learning approaches for both parcel-wise feature extraction and temporal modeling should be investigated.

\section{Acknowledgments}

This work is supported by the French National Research Agency under the grant ANR-18-CE23-0023. The authors want to thank the ASP (French payment agency) and the French agricultural ministry for the rich discussions and their guidance for the nomenclature choice and crop rotation schemes.

\section{References}

Aurdal, L., R. B. Huseby, L. Eikvil, R. Solberg, D. Vikhamar and A. Solberg. 2005. Use of hidden Markov models and phenology for multitemporal satellite image classification: Applications to mountain vegetation classification. Pages 16-18 in Proceedings of International Workshop Analysis Multi-Temporal Remote Sensing Images.

Belgiu, M. and O. Csillik. 2018. Sentinel-2 cropland mapping using pixel-based and object-based time-weighted dynamic time warping analysis. Remote Sensing of Environment 204:509-523.

Berzsenyi, Z., B. Györffy and D. Q. Lap. 2000. Effect of crop rotation and fertilisation on maize and wheat yields and yield stability in a long-term experiment. European Journal of Agronomy 13 (2):225-244.

Boryan, C., Z. Yang, R. Mueller and M. Craig. 2011. Monitoring us agriculture: The US department of agriculture, national agricultural statistics service, cropland data layer program. Geocarto International 26 (5):341-358.

Castellazzi, M. S., G. A. Wood, P. J. Burgess, J. Morris, K. F. Conrad and J. N. Perry. 2008. A systematic representation of crop rotations. Agricultural Systems 97 (1):26-33.

Defourny, P., S. Bontemps, N. Bellemans, C. Cara, G. Dedieu, E. Guzzonato, O. Hagolle, J. Inglada, L.-t. Nicola, T. Rabaute, M. Savinaud, C. Udroiu, S. Valero, A. Bégué, J.-F. Dejoux, A. El Harti, J. Ezzahar, N. Kussul, K. Labbassi, V. Lebourgeois, Z. Miao, T. Newby, A. Nyamugama, N. Salh, A. Shelestov, V. Simonneaux, P.S. Traore, S.S. Traore and B. Koetz. 2019. Near real-time agriculture monitoring at national scale at parcel resolution: Performance assessment of the Sen2-agri automated system in various cropping systems around the world. Remote Sensing of Environment 221:551-568.

Deledalle, C.-A., L. Denis, F. Tupin, A. Reigber and M. Jäger. 2015. NL-SAR: A unified nonlocal framework for resolution-preserving (pol)(in) SAR denoising. IEEE Transactions on Geoscience and Remote Sensing 53 (4):2021-2038. 
European Commission. 2016. Towards future Copernicus service components in support to agriculture? <https://ec.europa.eu/ jrc/sites/jrcsh/files/Copernicus_concept_note_agriculture.pdf> Accessed on 7 May 2020.

GISAT s.r.o. 2018. Sentinel-2 Agriculture: Czech Agriculture National Demonstrator (CzechAgri) Final Report. <http://www.esasen2agri.org/wp-content/uploads/docs/CzechAgri\%20Final\%20 Report\%201.2.pdf> Accessed on 7 May 2020.

Hagolle, O., M. Huc, D. Villa Pascual and G. Dedieu. 2010. A multitemporal method for cloud detection, applied to Formosat-2, Ven $\mu$ s, Landsat and Sentinel-2 images. Remote Sensing of Environment 114 (8):1747-1755.

Hagolle, O., M. Huc, D. Villa Pascual and G. Dedieu. 2015. A multi-temporal and multi-spectral method to estimate aerosol optical thickness over land, for the atmospheric correction of formosat-2, Landsat, Ven $\mu$ s and Sentinel-2 images. Remote Sensing 7 (3):2668-2691.

Hoberg, T., F. Rottensteiner, R. Q. Feitosa, and C. Heipke. 2015. Conditional random fields for multitemporal and multiscale classification of optical satellite imagery. IEEE Transactions on Geoscience and Remote Sensing 53 (2):659-673.

Inglada, Jordi. 2016. OTB Gapfilling, a temporal gapfilling for image time series library, Zenodo. <http://doi.org/10.5281/ zenodo.45572>.

Inglada, J., M. Arias, B. Tardy, O. Hagolle, S. Valero, D. Morin, G. Dedieu, G. Sepulcre, S. Bontemps and P. Defourny. 2015. Assessment of an operational system for crop type map production using high temporal and spatial resolution satellite optical imagery. Remote Sensing 7 (9):12356-12379.

Karlen, D. L., E. G. Hurley, S. S. Andrews, C. A. Cambardella, D. W. Meek, M. D. Duffy and A. P. Mallarino. 2006. Crop rotation effects on soil quality at three northern corn/soybean belt locations. Agronomy Journal 98 (3):484-495.

Kenduiywoa, B. K., D. Bargiel and U. Soergel. 2015. Spatial-temporal conditional random fields crop classification from Terrasar-X images. ISPRS Annals of the Photogrammetry, Remote Sensing and Spatial Information Sciences 2 (3):79.

Kussul, N., G. Lemoine, F. J. Gallego, S. V. Skakun, M. Lavreniuk and A. Y. Shelestov. 2016. Parcel-based crop classification in Ukraine using Landsat-8 data and Sentinel-1a data. IEEE Journal of Selected Topics in Applied Earth Observations and Remote Sensing 9 (6):2500-2508.

Lee, J.-S. 1980. Digital image enhancement and noise filtering by use of local statistics. IEEE Transactions on Pattern Analysis and Machine Intelligence (2):165-168.

Leite, P.B.C., R. Q. Feitosa, A. R. Formaggio, G. A. da Costa, O. Pedro, K. Pakzad and I. Del'Arco Sanches. 2011. Hidden Markov models for crop recognition in remote sensing image sequences. Pattern Recognition Letters 32 (1):19-26.
Li, H., C. Zhang, S. Zhang and P. M. Atkinson. 2019. Full year crop monitoring and separability assessment with fully-polarimetric L-band UAVSAR: A case study in the Sacramento Valley, California. International Journal of Applied Earth Observation and Geoinformation 74:45-56.

Liu, D., K. Song, J.R.G. Townshend and P. Gong. 2008. Using local transition probability models in Markov random fields for forest change detection. Remote Sensing of Environment 112 (5):22222231.

Manning, C. D., P. Raghavan and H. Schütze. 2008. Introduction to Information Retrieval, vol. 1 (1). Cambridge, England: Cambridge University Press.

Osman, J., J. Inglada and J.-F. Dejoux. 2015. Assessment of a Markov logic model of crop rotations for early crop mapping. Computers and Electronics in Agriculture 113:234-243.

Ottosen, T.-B.ø, S.T.E. Lommen and C. A. Skjãÿth. 2019. Remote sensing of cropping practice in northern Italy using time-series from sentinel-2. Computers and Electronics in Agriculture 157:232-238.

Palchowdhuri, Y., R. Valcarce-Di eiro, P. King and M. Sanabria-Soto. 2018. Classification of multi-temporal spectral indices for crop type mapping: A case study in Coalville, UK. The Journal of Agricultural Science 156 (1):24-36.

Roscher, R., B. Waske and W. Förstner. 2017. Kernel discriminative random fields for land cover classification. Pages 1-5 in Proceedings of IAPR Workshop on Pattern Recognition in Remote Sensing (PRRS).

Sen2-Agri. Czech agriculture national demonstrator - final report, 2018. <http://www.esa-sen2agri.org/wp-content/uploads/ docs/CzechAgri\%20Final\%20Report\%201.2.pdf> Accessed on February 6, 2018

Siachalou, S.u, G. Mallinis and M. Tsakiri-Strati. 2015. A hidden Markov models approach for crop classification: Linking crop phenology to time series of multi-sensor remote sensing data. Remote Sensing, 7 (4): 3633-3650.

Veloso, A., S. Mermoz, A. Bouvet, T. Le Toan, M. Planells, J.-F. Dejoux and E. Ceschia. 2017. Understanding the temporal behavior of crops using Sentinel-1 and Sentinel-2-like data for agricultural applications. Remote Sensing of Environment 199:415-426.

Vuolo, F., M. Neuwirth, M. Immitzer, C. Atzberger, and W.-T. Ng. 2018. How much does multi-temporal Sentinel-2 data improve crop type classification? International Journal of Applied Earth Observation and Geoinformation 72:122-130.

Wagner, B. N. and B. Waske. 2018. Combining Sentinel-1 and Sentinel-2 data for improved land use and land cover mapping of monsoon regions. International Journal of Applied Earth Observation and Geoinformation 73:595-604.

Whelen, T. and P. Siqueira. 2017. Use of time-series L-band UAVSAR data for the classification of agricultural fields in the San Joaquin Valley. Remote Sensing of Environment 193:216-224. 